\title{
Initiation of insulin therapy in patients with type 2 diabetes: An observational study
}

\author{
SPELA ZEROVNIK \\ MITJA KOS \\ IGOR LOCATELLI* \\ University of Ljubljana \\ Faculty of Pharmacy \\ Ljubljana, Slovenia
}

The aim of the study was to assess the initiation of insulin therapy in patients with type 2 diabetes using health claims data on prescription medicines. The study evaluated time to insulin initiation and prescribing patterns of other antidiabetic medicines before and after insulin initiation. Five years after starting non-insulin antidiabetic therapy, $6.4 \%$ of patients were prescribed insulin, which is substantially lower compared to other similar studies. Among all patients who initiated insulin therapy in 2013, $30 \%$ did not continue any other antidiabetic therapy. However, this proportion was lowered to $20 \%$ in 2018. Before insulin initiation in 2018, metformin was prescribed in only $67 \%$ of patients and sulfonylureas in $78 \%$ of patients. Moreover, metformin and sulfonylureas were discontinued after insulin initiation in 26 and $37 \%$ of patients, resp. More attention should be paid to the continuation of oral antidiabetics, particularly metformin, after insulin initiation.

Keywords: diabetes mellitus, insulin, metformin, sulfonylurea

According to international (1) and Slovenian guidelines (2), metformin is recommended as first-line therapy in patients with type 2 diabetes (T2D) unless contraindicated or not well tolerated. If the target glycated haemoglobin level $(<7 \%$ for most patients) cannot be achieved with metformin monotherapy, stepwise addition of sulfonylureas or other antidiabetic medicines, depending on the patient's comorbidities, is recommended $(1,2)$. Most patients with T2D, especially those with a longer history of diabetes, will eventually require insulin therapy (3). A recent study (4) showed that initiation of insulin therapy in Slovenia and other countries in Central and South-Eastern Europe is often delayed, which may result in poorer outcomes and reduced quality of life in patients with T2D (5). However, it is not known how quickly insulin therapy is initiated in newly treated patients with T2D (de novo patients) after the start of non-insulin antidiabetic therapy. In addition, little is known about the continuation of oral antidiabetic medicines and glucagon-like peptide-1 (GLP-1) analogues after the initiation of insulin.

\footnotetext{
*Correspondence; e-mail address: Igor.Locatelli@ffa.uni-lj.si
} 
Therefore, the aim of this study was to describe the initiation of insulin therapy in patients with T2D, especially to evaluate the time to insulin initiation in de novo patients with T2D, and to assess the prescribing patterns of non-insulin antidiabetic medicines before and after insulin initiation.

\section{EXPERIMENTAL}

This nationwide pharmacoepidemiological study was based on health claims data on prescription medicines for the period 2009-2018. Three different cohorts of patients were used to evaluate the initiation of insulin therapy in patients with T2D. The first cohort was formed to assess the time of insulin initiation in de novo patients who started with noninsulin antidiabetic therapy. In addition, we formed two cohorts (two different time periods) of all patients already treated with non-insulin antidiabetic therapy to compare non-insulin antidiabetic therapy before and after insulin initiation.

The study was approved by the National Medical Ethics Committee (Komisija RS za medicinsko etiko, Ministrstvo za zdravje, Štefanova 5, 1000 Ljubljana; registration number: 0120-264/2019/5)

\section{Data sources}

The Slovenian healthcare system is based on the Bismarck model and provides compulsory health insurance for the entire Slovenian population of 2 million. The healthcare fund is managed by the Health Insurance Institute of Slovenia (HIIS), which acts as a healthcare decision-maker and payer (6). Some healthcare services (including some groups of medicines) are fully covered by HIIS, while others are partially covered by complementary voluntary health insurance. All antidiabetic medicines are fully covered by compulsory health insurance.

An Outpatient Prescription Medicines Database used in this study was obtained from the National Institute of Public Health (NIJZ). The database includes data on all dispensed outpatient prescriptions for the entire Slovenian population. It contains information on dispensed medicines coded using Anatomical Therapeutic Chemical (ATC) classification, date of prescription, amount of medicine dispensed, and information about the patient (including gender, year of birth and geographical region). Patients can be followed within the database using unique anonymous patient identifiers. Because one cohort of patients in this study was followed over a longer time period, we also used information on patient death. This information was obtained from Causes of Death data that includes the month and year of death and the primary cause of death coded using the International Classification of Diseases $\left(10^{\text {th }}\right.$ revision).

\section{Selection of de novo patients starting antidiabetic therapy in 2012}

De novo patients starting antidiabetic therapy in 2012 were defined as patients who received a prescription for antidiabetic medicine (ATC code: A10) in 2012 and had not received a prescription for an antidiabetic medicine in the three years prior to the date of the first prescription in 2012 (incident cases). 
These patients were divided into two groups: patients who started insulin therapy [patients who received one or more prescriptions for insulin (ATC code: A10A) within 30 days of the first dispensed prescription for antidiabetic medicine] and patients who started non-insulin antidiabetic therapy. Descriptive statistics were used to describe patients who started insulin therapy, whereas patients who started non-insulin antidiabetic therapy were included in cohort $\mathrm{A}$ and followed until insulin initiation. Patients under 45 years of age were further excluded to minimize the inclusion of patients with early-onset T2D in cohort A. The date of entry into cohort A was defined as the date of the first dispensed prescription for antidiabetic medicine. Baseline antidiabetic therapy was assessed on and within 30 days of the date of entry into cohort A.

\section{Selection of patients being treated with non-insulin antidiabetic therapy (cohorts B and C)}

In addition, the initiation of insulin therapy in patients who were already treated with non-insulin antidiabetic therapy was assessed. Two cohorts of patients were derived to cover two different time periods. Patients who were on non-insulin antidiabetic therapy on June 30, 2012, were included in cohort B, and patients who were on non-insulin antidiabetic therapy on June 30, 2017, were included in cohort C. Patients included in these two cohorts filled at least one prescription for an antidiabetic medicine within one year before the start of the follow-up. Patients who received one or more prescriptions for insulin during this period and patients under 45 years of age were excluded. Patients from both cohorts were followed for one year.

\section{Description of antidiabetic therapy at insulin initiation}

The type of insulin prescribed at the initiation of insulin therapy and the non-insulin antidiabetic therapy that was prescribed before and after insulin initiation were identified. The following ATC codes were used to define the type of insulin: (i) basal insulins (A10AC, A10AE), (ii) biphasic insulins (A10AD) and (iii) fast-acting insulins (A10AB). Antidiabetic therapy before insulin initiation was evaluated during the 135 days preceding the first date of insulin dispensing, and antidiabetic therapy after insulin initiation was assessed at the date of insulin initiation or within the subsequent 135 days. A 135-day period was used because all antidiabetic medicines are usually dispensed for a 3-month supply, to which a 45-day gap period was added. The continuation of metformin and sulfonylurea therapy after insulin initiation was assessed in a group of patients who received at least one prescription for metformin/sulfonylurea within the 135 days before starting insulin therapy. These patients were considered to remain on metformin/sulfonylurea therapy if they received at least one prescription for metformin/sulfonylurea on the date of insulin initiation or during the next 135 days.

\section{Statistical analysis}

Time to insulin initiation was calculated for de novo patients who started non-insulin antidiabetic therapy in 2012 (cohort A) using the Kaplan-Meier survival function. Patients from cohort A were followed from the date of their first dispensed prescription for noninsulin antidiabetic medicine (date of entry into cohort A) to the date of their first dispensed prescription for insulin (event date) or to the last day of the month in which they 
died (censored data) or to June 30, 2018 (also censored data). A Cox proportional regression model was developed to evaluate the association between time of insulin initiation and patient age and gender. In addition, the probability of initiating insulin within 10 years of starting non-insulin antidiabetic therapy was estimated by extrapolating the survival curve using exponential and Weibull functions. All analyses were conducted using IBM SPSS Statistics for Windows, Version 27.0 (Armonk, USA).

\section{RESULTS AND DISCUSSION}

\section{Characteristics of de novo patients starting insulin therapy in 2012}

In 2012, 9,596 patients started antidiabetic therapy of whom 1,203 (12.5\%) started insulin therapy. 1,027 (85.4\%) of these patients started insulin monotherapy, whereas the others started insulin therapy in combination with oral antidiabetic medicines or GLP-1 analogues. The mean age of patients starting insulin therapy was 47.1 years [standard deviation (SD): 20.4], and the majority of them were female $(n=807 ; 67.1 \%)$. Fig. 1 shows the age distribution of patients who started insulin therapy separately for males $(n=396)$ and females $(n=807)$.

There was a large variation in the age distribution between male and female patients. The majority of male patients who started insulin therapy were at least 45 years of age $(n=316 ; 79.8 \%)$, whereas the majority of female patients who started insulin therapy were $20-44$ years old $(n=517 ; 64.1 \%)$. Of the 8,036 de novo patients who were at least 45 years of age and started antidiabetic therapy in 2012, 567 (7.1\%) started insulin therapy, with the majority of them receiving insulin monotherapy (73.2 \%). The proportions of female and male patients at least 45 years of age who started insulin therapy were similar.

The majority of female patients in the 20-44-year-old group were probably prescribed insulin for gestational diabetes. Insulin is the treatment of choice for patients with gestational diabetes in whom non-pharmacological measures provide insufficient glycaemic control (7). In contrast, the majority of patients at least 45 years of age likely started insulin
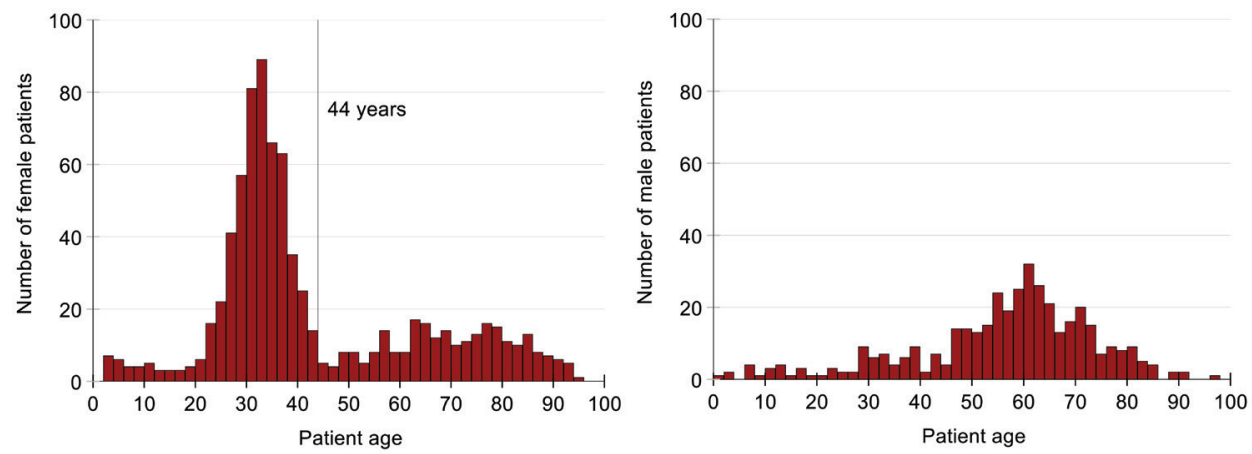

Fig. 1. Age distribution of de novo patients starting insulin therapy in 2012. Left panel: female patients $(n=807)$, right panel: male patients $(n=396)$. 
therapy due to T2D. The proportion of patients with T2D who started insulin as first-line therapy (as monotherapy or in combination with oral antidiabetic medicines or GLP-1 analogues) was similar to the study by Montvida et al. in the USA (8) in which $8-10 \%$ of patients with T2D aged 18-80 years started insulin as first-line therapy. However, two studies based on UK electronic health records for the period 2000-2017 showed a much lower proportion of patients with T2D initiating insulin as first-line therapy compared to our study $(9,10)$. According to clinical guidelines, insulin can be considered for first-line T2D therapy in patients with severe or symptomatic hyperglycaemia at the time of diagnosis $(1,2)$. However, as data on glycated haemoglobin (HbA1c) levels were not available in either the UK studies $(9,10)$ or our study, we cannot assess the rationality for initiating insulin as first-line therapy in these studies. It is possible that there is better screening for T2D in the UK, or that in Slovenia insulin is initiated as first-line therapy in T2D patients at a lower HbA1c threshold than in the UK.

\section{Characteristics of patients who started non-insulin antidiabetic therapy in 2012 (cohort} A) and time to insulin initiation

Out of the 8,393 patients who started non-insulin antidiabetic therapy in 2012, 924 $(11.0 \%)$ were younger than 45 years and were excluded, resulting in 7,469 patients in cohort A. The mean patient age was 65 years (SD: 11) and 3,388 (45.4\%) were women. The majority of patients started with metformin monotherapy (59.5\%), with fewer patients starting with sulfonylurea monotherapy (19.4\%) or metformin in combination with sulfonylureas $(15.5 \%)$. During a mean follow-up of 5.5 years after initiation of non-insulin antidiabetic therapy, 543 patients $(7.3 \%)$ started insulin therapy. The Kaplan-Meier estimate of the 1-year probability of starting insulin therapy was $1.2 \%$ [95 \% confidence interval (CI): 1.0-1.5\%], and the 5-year probability was $6.4 \%$ (95\% CI: 5.8-7.0\%). According to the survival function extrapolation, $12.7 \%$ (95 \% CI: 11.5-13.8 \%) of patients with T2D are expected to be prescribed insulin within 10 years of starting non-insulin antidiabetic therapy. Using Cox regression analysis, we found that patient age was inversely associated with the initiation of insulin therapy. The risk of insulin initiation decreased by $1 \%$ with each increasing year of age [hazard ratio $(\mathrm{HR})=0.990 ; 95 \% \mathrm{CI}$ : 0.982-0.999]. No difference in the risk of insulin initiation was found between male and female patients.

The proportion of patients who initiated insulin in the first 5 years and the extrapolated value to 10 years were much lower than the results of a study of Swedish patients with T2D, in which $25 \%$ of patients initiated insulin within 6 years and $42 \%$ within 10 years of their first oral antidiabetic medicine prescription (11). However, that study also included patients who were prescribed insulin on the same date as an oral antidiabetic medicine (3.3\% of patients). In our study, approximately $2 \%$ of patients at least 45 years of age received a prescription for insulin on the same day as the first prescription for a noninsulin antidiabetic medicine; however, these patients were not included in the time-to-event analysis. Another possible explanation for the higher proportion of patients initiating insulin is that insulins are usually introduced early in the course of T2D in Sweden. During 2006-2015, insulins were frequently prescribed as second-line therapy (12). In addition, the Swedish study (11) used healthcare records from 1993 to 2005, a period when novel antidiabetic medicines [GLP-1 analogues, dipeptidyl peptidase-4 (DPP-4) inhibitors and sodium-glucose co-transporter-2 (SGLT2) inhibitors] were not available. A study by 
Kostev et al. (13) also showed that the time to insulin initiation in Germany and the UK increased from 2005 to 2010, possibly due to the introduction of novel antidiabetic medicines. Another study of Dutch patients (14) also reported a higher proportion of patients with T2D at least 45 years of age starting insulin therapy during a 6 -year follow-up period (13.5\%). However, that study also included patients with T2D who were initially treated with insulin in combination with oral antidiabetic medicines or GLP-1 analogues.

In our study, insulin was initiated in a relatively small proportion of patients with T2D in the first five years after the start of non-insulin antidiabetic therapy. However, since we do not have data on HbA1c levels of patients, we cannot determine whether this proportion was lower because patients were well-controlled and had lower HbA1c levels or due to other reasons for delaying insulin initiation in Slovenia. A recent study (4) involving prescribers of antidiabetic medicines from five countries in Central and South-Eastern Europe showed that the majority of prescribers would not initiate insulin at an HbA1c threshold of 7-7.9 \%. The majority of prescribers from Slovenia would initiate insulin therapy at an $\mathrm{HbA1c}$ level of 8-8.9\%, while prescribers from other countries (Bulgaria, Croatia, Greece and Hungary) would initiate insulin at an even higher HbA1c level. The most important barrier to insulin initiation reported in that study was psychological distress.

\section{Characteristics of patients starting insulin therapy during follow-up}

The numbers of patients from each cohort that started insulin therapy during follow-up were 543 in cohort A, 2,198 in cohort B and 2,135 in cohort C. Their characteristics are presented in Table I. Patients who initiated insulin therapy were more frequently males. The most commonly prescribed antidiabetic medicines before insulin initiation were sulfonylureas (approximately 70-80 \%), followed by metformin (approximately 55-65 \%) and DPP-4 inhibitors. The proportion of patients who received metformin and sulfonylureas before insulin initiation was similar in all three cohorts. Patients in cohort $C$ received a higher number of different antidiabetic medicines before insulin initiation than patients in cohort B and were more frequently prescribed SGLT2 inhibitors and GLP-1 analogues.

\section{Type of insulin prescribed}

Table II shows the type of insulin prescribed at the initiation of insulin therapy and other non-insulin antidiabetic medicines prescribed after the initiation of insulin. The most commonly prescribed insulins at the initiation of insulin therapy were basal (especially intermediate-acting insulins), followed by biphasic insulins. According to international guidelines, basal insulins are the preferred initial insulin regimen in patients with T2D because of the lower risk of hypoglycaemia and weight gain compared to biphasic or prandial insulins (1). In the current study, intermediate-acting basal insulins were prescribed more frequently than long-acting basal insulins, which disagrees with the study by Wilke et al. (15), in which long-acting insulins were prescribed more commonly. Higher prescribing rates of intermediate-acting basal insulins in Slovenia compared to Germany might be due to Slovenian guidelines (2) that recommend intermediate-acting basal insulins over long-acting basal insulins and prescribing restrictions that apply to long-acting basal insulins due to their high prices. 


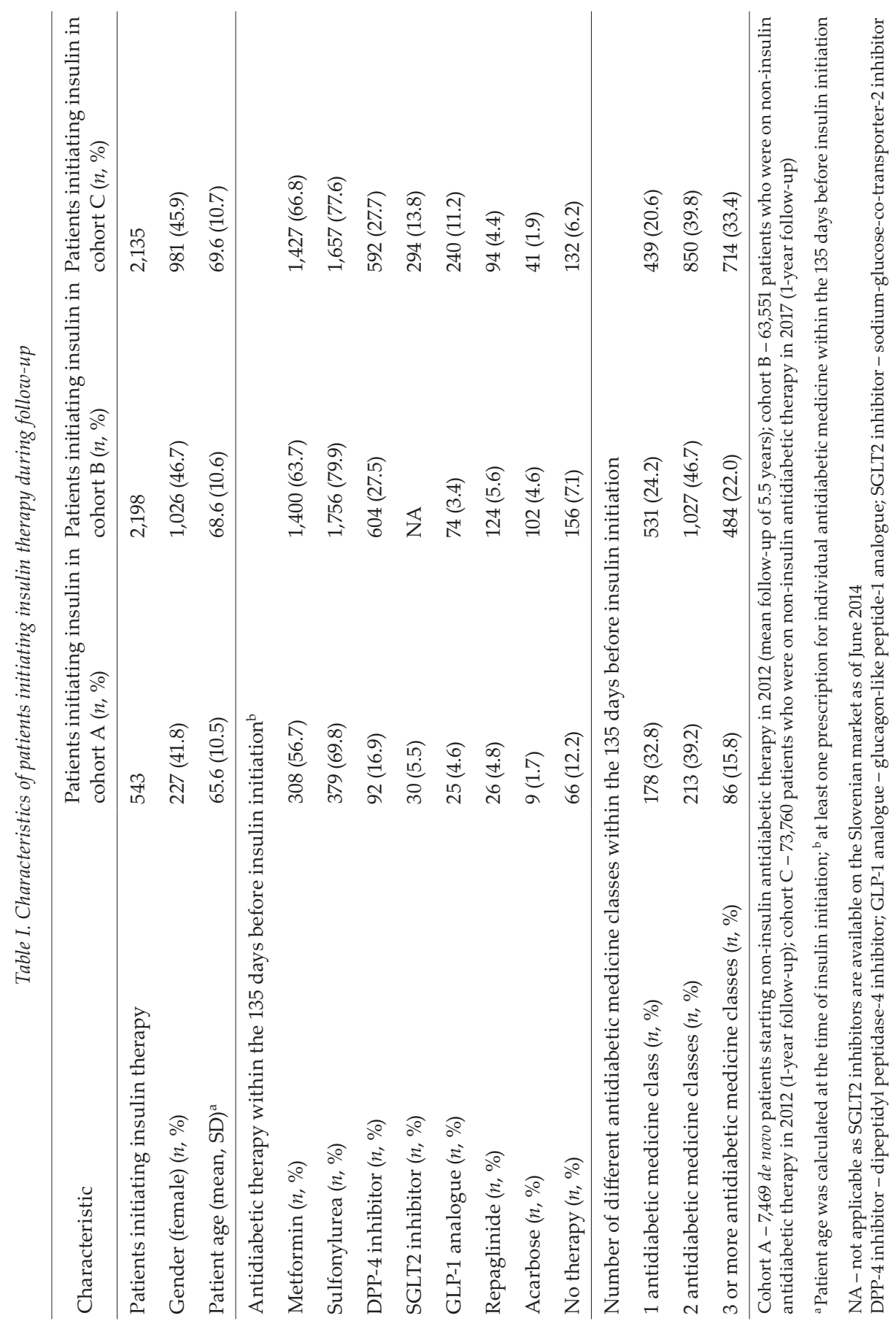


Table II. The type of insulin prescribed at the initiation of insulin therapy and non-insulin antidiabetic therapy after insulin initiation

\begin{tabular}{|c|c|c|c|}
\hline Type of insulin & $\begin{array}{l}\text { Patients } \\
\text { initiating insulin } \\
\text { in cohort A } \\
(n, \%)\end{array}$ & $\begin{array}{l}\text { Patients } \\
\text { initiating insulin } \\
\text { in cohort B } \\
(n, \%)\end{array}$ & $\begin{array}{l}\text { Patients } \\
\text { initiating insulin } \\
\text { in cohort C } \\
(n, \%)\end{array}$ \\
\hline Basal insulin & $219(40.3)$ & $1,020(46.4)$ & $1,192(55.8)$ \\
\hline Intermediate-acting insulin & $183(33.7)$ & $763(34.7)$ & $794(37.2)$ \\
\hline Long-acting insulin & $36(6.6)$ & $257(11.7)$ & $398(18.6)$ \\
\hline Biphasic insulin & $191(35.2)$ & $809(36.8)$ & $582(27.3)$ \\
\hline Basal and fast-acting insulin & $74(13.6)$ & $201(9.1)$ & $186(8.7)$ \\
\hline Intermediate-acting and fast-acting insulin & $63(11.6)$ & $99(4.5)$ & $138(6.5)$ \\
\hline Long-acting and fast-acting insulin & $11(2.0)$ & $102(4.6)$ & $48(2.2)$ \\
\hline Fast-acting insulin & $52(9.6)$ & $144(6.6)$ & $132(6.2)$ \\
\hline Other combinations & $7(1.3)$ & $24(1.1)$ & $43(2.0)$ \\
\hline \multicolumn{4}{|l|}{ Antidiabetic therapy after insulin initiation ${ }^{a}$} \\
\hline Insulin monotherapy & $192(35.4)$ & $649(30.4)$ & $435(20.4)$ \\
\hline $\begin{array}{l}\text { Insulin and metformin with or without } \\
\text { other AD (excluding SU) }\end{array}$ & $97(17.9)$ & $391(17.8)$ & $470(22.0)$ \\
\hline $\begin{array}{l}\text { Insulin and SU with or without other AD } \\
\text { (excluding metformin) }\end{array}$ & $98(18.0)$ & $378(17.2)$ & $408(19.1)$ \\
\hline $\begin{array}{l}\text { Insulin, metformin and SU with or } \\
\text { without other AD }\end{array}$ & $139(25.6)$ & $719(32.7)$ & $738(34.6)$ \\
\hline $\begin{array}{l}\text { Insulin and other } \mathrm{AD} \text { (excluding SU and } \\
\text { metformin) }\end{array}$ & 17 (3.1) & $61(2.9)$ & $84(3.9)$ \\
\hline Total number of patients & 543 & 2,198 & 2,135 \\
\hline
\end{tabular}

Cohort A - de novo patients starting non-insulin antidiabetic therapy in 2012; cohort B - patients who were on non-insulin antidiabetic therapy in 2012; cohort C - patients who were on non-insulin antidiabetic therapy in 2017

a Antidiabetic therapy was assessed at the date of insulin initiation and within the subsequent 135 days.

AD - antidiabetic, other AD - repaglinide, dipeptidyl peptidase- 4 inhibitor, glucagon-like peptide- 1 analogue, sodium-glucose co-transporter-2 inhibitor or acarbose, $\mathrm{SU}$ - sulfonylurea

ATC codes for insulins: basal insulins (A10AC, A10AE), biphasic insulins (A10AD), fast-acting insulins (A10AB).

\section{Antidiabetic therapy after insulin initiation}

A relatively high proportion of patients started taking insulin as monotherapy. However, the proportion of patients initiating insulin as monotherapy was higher in 2013 (30\%) than in 2018 (20\%) (Table II), which is encouraging. A study by Wilke et al. (15) found an even higher proportion of insulin monotherapy (approximately $40 \%$ ) in German patients with T2D who started insulin between 2013 and 2015. 
The most commonly prescribed oral antidiabetic medicines after insulin initiation were metformin and sulfonylureas, which is consistent with other studies $(16,17)$. Approximately one-third of patients received both metformin and sulfonylurea with or without other antidiabetic medicines. In addition, the proportions of patients receiving metformin or sulfonylureas after starting insulin therapy were similar (approximately $50 \%$ for both medicines, Table II). After starting insulin therapy, metformin was discontinued in 33, 31 and $26 \%$ of patients from cohorts A, B, and C, resp. Although the proportion of patients who continued metformin therapy increased from 69 to $74 \%$ between 2013 and 2018, it was still lower than in the study by Xu et al. (16), who found that almost $85 \%$ of US patients with T2D continued metformin after initiating insulin. In addition, according to clinical guidelines $(1,2)$, metformin therapy should be continued after the initiation of insulin. On the other hand, sulfonylureas were discontinued in 46,44 and $37 \%$ of patients from cohorts A, B and C, resp. These proportions are higher compared with two studies in US patients with T2D, in which sulfonylureas were discontinued in approximately $30 \%$ of patients within 90 days of starting insulin $(16,18)$. According to guidelines, the dose of sulfonylureas should be reduced by $50 \%$ or they should be discontinued after the initiation of insulin (1).

This nationwide study describes the initiation of insulin therapy in patients with T2D in three large cohorts of patients in different time periods. To the best of our knowledge, this is the first European study that evaluated the discontinuation of metformin and sulfonylureas after the initiation of insulin therapy. However, interpreting the results of this study must be done in the context of its limitations. First, patient diagnoses are not included in the database used in this study. Therefore, the definition of patients with T2D was based on the dispensed prescription for antidiabetic medicine and patient age (excluding patients treated with insulin at the time of cohort entry and patients under 45 years of age). Second, because we did not have data on HbA1c levels, we could not determine whether patients were introduced insulin in a timely manner. Having these data would allow us to determine the extent of clinical inertia in insulin initiation in Slovenia, which was partially addressed in a study by Campbell et al. (4). A high prevalence of clinical inertia regarding insulin initiation has already been found in some European countries (19).

\section{CONCLUSIONS}

The majority of patients who started non-insulin antidiabetic therapy were treated with metformin as monotherapy, or in combination with sulfonylurea. Approximately $7 \%$ of patients with T2D were prescribed insulin within 5 years of initiating non-insulin antidiabetic therapy, which is lower than the data in the existing literature. A surprisingly high proportion of patients who started taking insulin in our study did so as monotherapy, which is not in line with clinical guidelines. However, the proportion of patients starting insulin as monotherapy in 2018 (30\%) was lower than in 2013 (20\%). The high proportion of insulin monotherapy is due to high rates of discontinuing oral antidiabetic therapy after initiating insulin. After insulin initiation in 2018, metformin and sulfonylureas were discontinued in about 30 and $40 \%$ of patients, resp. Further studies are needed to evaluate how prescribing patterns of insulin and non-insulin antidiabetic medicines after insulin initiation affect patient outcomes and healthcare expenditure. 


\section{REFERENCES}

1. M. J. Davies, D. A. D'Alessio, J. Fradkin, W. N. Kernan, C. Mathieu, G. Mingrone, P. Rossing, A. Tsapas, D. J. Wexler and J. B. Buse, Management of hyperglycemia in type 2 diabetes, 2018. A consensus report by the American Diabetes Association (ADA) and the European Association for the Study of Diabetes (EASD), Diabetes Care 41 (2018) 2669-2701; https://doi.org/10.2337/dci18-0033

2. Slovenian Endocrinology Association, Slovenian Guidelines on Type 2 Diabetes Management 2016 [in Slovene]; https://endodiab.si/priporocila/smernice-za-vodenje-sladkorne-bolezni/; last access date November 30, 2020.

3. P. Home, M. Riddle, W. T. Cefalu, C. J. Bailey, R. G. Bretzel, S. Del Prato, D. Leroith, G. Schernthaner, L. van Gaal and I. Raz, Insulin therapy in people with type 2 diabetes: opportunities and challenges? Diabetes Care 37 (2014) 1499-1508; https://doi.org/10.2337/dc13-2743

4. M. D. Campbell, D. Babic, U. Bolcina, L. Smirčić-Duvnjak, T. Tankova, A. Mitrakou, P. Kempler and A. Janez, High level of clinical inertia in insulin initiation in type 2 diabetes across Central and South-Eastern Europe: insights from SITIP study, Acta Diabetol. 56 (2019) 1045-1049; https:// doi.org/10.1007/s00592-019-01346-1

5. G. Goodall, E. M. Sarpong, C. Hayes and W. J. Valentine, The consequences of delaying insulin initiation in UK type 2 diabetes patients failing oral hyperglycaemic agents: a modelling study, BMC Endocr. Disord. 9 (2009) Article ID 19 (9 pages); https://doi.org/10.1186/1472-6823-9-19

6. T. Albreht, R. Pribaković Brinovec, D. Jošar, M. Poldrugovac, T. Kostnapfel, M. Zaletel, D. Panteli and A. Maresso, Slovenia: Health system review, Health Syst. Trans. 18 (2016) (207 pages); https:// www.euro.who.int/_data/assets/pdf_file/0018/312147/HiT-Slovenia_rev3.pdf; last access date February 15, 2021.

7. E. Chiefari, B. Arcidiacono, D. Foti and A. Brunetti, Gestational diabetes mellitus: an updated overview, J. Endocrinol. Invest. 40 (2017) 899-909; https://doi.org/10.1007/s40618-016-0607-5

8. O. Montvida, J. Shaw, J. J. Atherton, F. Stringer and S. K. Paul, Long-term trends in antidiabetes drug usage in the U.S.: Real-world evidence in patients newly diagnosed with type 2 diabetes, Diabetes Care 41 (2018) 69-78; https://doi.org/10.2337/dc17-1414

9. S. Wilkinson, I. Douglas, H. Stirnadel-Farrant, D. Fogarty, A. Pokrajac, L. Smeeth and L. Tomlinson, Changing use of antidiabetic drugs in the UK: trends in prescribing 2000-2017, BMJ Open 8 (2018) e022768; https://doi.org/10.1136/bmjopen-2018-022768

10. M. Sharma, I. Nazareth and I. Petersen, Trends in incidence, prevalence and prescribing in type 2 diabetes mellitus between 2000 and 2013 in primary care: a retrospective cohort study, BMJ Open 6 (2016) e010210; https://doi.org/10.1136/bmjopen-2015-010210

11. A. Ringborg, P. Lindgren, D. D. Yin, M. Martinell and J. Stalhammar, Time to insulin treatment and factors associated with insulin prescription in Swedish patients with type 2 diabetes, Diabetes Metab. 36 (2010) 198-203; https://doi.org/10.1016/j.diabet.2009.11.006

12. F. Persson, J. Bodegard, J. T. Lahtela, T. Nystrom, M. E. Jorgensen, M. L. Jensen, H. L. Gulseth, M. Thuresson, F. Hoti, D. Nathanson, A. Norhammar, K. I. Birkeland, J. G. Eriksson and J. W. Eriksson, Different patterns of second-line treatment in type 2 diabetes after metformin monotherapy in Denmark, Finland, Norway and Sweden (D360 Nordic): A multinational observational study, Endocrinol. Diabetes Metab. 1 (2018) e00036; https://doi.org/10.1002/edm2.36

13. K. Kostev and W. Rathmann, Changes in time to insulin initiation in type 2 diabetes patients: a retrospective database analysis in Germany and UK (2005-2010), Prim. Care Diabetes 7 (2013) 229_ 233; https://doi.org/10.1016/j.pcd.2013.03.003

14. J. M. van den Heuvel, N. Farzan, M. van Hoek, A.-H. Maitland-van der Zee and F. Ahmadizar, Mining treatment patterns of glucose-lowering medications for type 2 diabetes in the Netherlands, BMJ Open Diabetes Res. Care 8 (2020) e000767; https://doi.org/10.1136/bmjdrc-2019-000767 
15. T. Wilke, N. Picker, S. Mueller, S. Geier, J. Foersch, J. Aberle, S. Martin, M. Riedl and M. Gabler, Real-world insulin therapy in German type 2 diabetes mellitus patients: patient characteristics, treatment patterns, and insulin dosage, Diabetes Metab. Syndr. Obes. 12 (2019) 1225-1237; https://doi. org/10.2147/DMSO.S214288

16. Y. Xu, S. J. Pilla, G. C. Alexander and I. B. Murimi, Use of non-insulin diabetes medicines after insulin initiation: A retrospective cohort study, PLoS One 14 (2019) e0211820; https://doi.org/10.1371/ journal.pone.0211820

17. K. Khunti, T. Damci, L. Meneghini, C. Y. Pan and J.-F. Yale, Study of once daily levemir (SOLVE $\left.{ }^{\mathrm{TM}}\right)$ : insights into the timing of insulin initiation in people with poorly controlled type 2 diabetes in routine clinical practice, Diabetes Obes. Metab. 14 (2012) 654-661; https://doi. org/10.1111/j.1463-1326.2012.01602.x

18. D. C. Beachler, G. Fernandes, G. Deshpande, J. Jemison, L. G. Lyons, S. Lanes, J. Liu and A. McNeill, Patient and prescriber characteristics among patients with type 2 diabetes mellitus continuing or discontinuing sulfonylureas following insulin initiation: data from a large commercial database, Curr. Med. Res. Opin. 34 (2018) 1061-1069; https://doi.org/10.1080/03007995.2017.1416348

19. K. Khunti, M. B. Gomes, S. Pocock, M. V. Shestakova, S. Pintat, P. Fenici, N. Hammar and J. Medina, Therapeutic inertia in the treatment of hyperglycaemia in patients with type 2 diabetes: A systematic review, Diabetes Obes. Metab. 20 (2018) 427-437; https://doi.org/10.1111/dom.13088 